\title{
A comparison of obturation techniques
}

Comparative study of two canal obturation techniques by image analysis and EDS microanalysis.

M. Guigand, D. Glez, E. Sibayan, G. Cathelineau and J-M. Vulcain Br Dent J 2005; 198: 707-711

Aim

The aim of this in vitro study was to compare the marginal adaptation achieved by two obturation techniques (lateral condensation and Thermafil) using human teeth prepared by continuous rotation with the HERO $642{ }^{\circledR}$ system.

Method

The percentages of gaps and sealer on the root canal surface were determined by analysing the images of 12 sections per tooth. Tubule sealer penetration was assessed by backscattered scanning electron microscopy and energy dispersive spectrometry microanalysis.

Results

The Thermafil obturation technique resulted in virtually no gaps and very low amounts of sealer on the root surface, unlike the lateral condensation technique. Tubule sealer penetration occurred with both techniques, but was deeper, especially in the mid and apical zones, with the lateral condensation technique.

\section{IN BRIEF}

- This study shows that the Thermafil technique provides better results than cold lateral condensation if a corono-apical tapered preparation is made.

- This technique may be used even if the apical part of the root canal is instrumented with .02 taper files.

- The practitioner has to take into account the different tapers of both Thermafil and apical file by using a smaller diameter for the Thermafil obturator.

\section{COMMENT}

The success of endodontic treatment can be attributed to the root canal preparation associated with chemical irrigation and the endodontic filling providing a tight seal, which is the only way to ensure a long-lasting result.

According to Ingle and Dow, the hermeticity of the root canal filling plays a key role by promoting the healing process. This study highlights the superiority of the Thermafil obturation technique with respect to cold lateral condensation which remains the 'gold standard'.

In this study, the root canal preparation and irrigation parameters were clearly defined (in a clinical way), so interferences upon the results have been avoided. The image analysis of sections enables determination of gaps for each level of the root canal whichever technique was used.

The results are widely in favour of the Thermafil Technique where gaps were 12 times less numerous. In the same way, the sealer surface appears to be five times larger for the cold lateral condensation, suggesting a lesser hermeticity, although deeper impregnation of dentinal tubules is observed. This is surely due to the pressure exerted by the spreader during the cold lateral condensation. Actually, whatever the level, the less sealer there is, the more hermetic the filling is.

This study highlights that in itself, the root canal filling technique may influence the result independently from the root canal preparation and irrigation.

J. M. Vulcain, Head of Odontology Department, C.H.U. of Rennes, France

doi: 10.1038/sj.bdj.4812390 